\title{
A State of Charge Estimation of The Lithium-Ion Batteries Based on Reduced-Order Unscented Kalman Filtering Method
}

\author{
Emmanuel Appiah ${ }^{1}$, Shunli Wang ${ }^{1}$, Chuanyun Zou ${ }^{1}$, Bobobee Etse Dablu ${ }^{1}$, Takyi-Aninakwa Paul ${ }^{1}$, Haque Md \\ Amdadul $^{1}$, Carlos Fernandez ${ }^{2}$
}

1. School of Information Engineering, Southwest University of Science and Technology, Mianyang 621010, China;

2. School of Pharmacy and Life Sciences, Robert Gordon University, Aberdeen AB10-7GJ, UK.

Corresponding author: Emmanuel Appiah. Tel.: +86-17709050144. E-mail address: bikusnas.25@gmail.com

The work is supported by the National Natural Science Foundation of China (No. 61801407), Sichuan Science and Technology program (No. 2019YFG0427), China Scholarship Council (No. 201908515099), and Fund of

Robot Technology Used for Special Environment Key Laboratory of Sichuan Province (No. 18kftk03).

\begin{abstract}
:
State of Charge (SOC) represents the available battery capacity and is one of the most important states that need to be monitored to optimize the performance and extend the lifetime of lithium-ion batteries. SOC estimation is a challenging task hindered by considerable changes in battery characteristics over its lifetime due to aging and distinct nonlinear behavior. This paper compares two of the basic methods and algorithms for SOC estimation of lithium-ion batteries (LIBs) focusing on the description of the two techniques in a test experiment and the elaboration of their differences for use in battery management systems (BMS) applications. A Reduced-order unscented Kalman filter method is used for estimation and tracking to realizer real-time high-precision estimation of lithium-ion battery state of charge. Experimental tests are carried out with a lithium-ion battery cell for model and state estimation validations. Many researchers have proposed different methods of estimating SOC that raised the challenge of establishing a relationship between the accuracy and robustness of the method. The experimental results of the OCV-SOC estimation method, Hybrid Pulse Power Characterization (HPPC) test, and Beijing Bus Dynamic Stress Test (BBDST) working condition method are analyzed. The error of SOC estimation based on the established Thevenin RC modeling using the Reduced-order-unscented Kalman filter is less than $0.3 \%$. The result from the use of the reduced-order unscented Kalman filtering algorithm proves that it has high accuracy in the state of charge estimation of the lithium-ion batteries.
\end{abstract}

Keywords: lithium-ion battery, open-circuit voltage, state of charge estimation, battery management system, HPPC test, reduced-order unscented Kalman filter

DOI: $10.7176 /$ JETP/11-6-05

Publication date: December $30^{\text {th }} 2021$

\section{Introduction}

The lithium-ion battery (LIB) is perhaps the most used battery among the new energy sources and is more likely to replace lead-acid batteries. Interest in developing batteries for electric vehicles and mobile applications has increased tremendously because of its clean energy and environmental friendliness. Lithium-ion batteries are now widely used as a main source of energy for many applications from portable electronics to electric vehicles (EVs) $[1,2]$. The most promising battery technologies are the LIBs and are the preferred power supply for EVs, laptops, notebooks, mobile phones, tablets, rechargeable lamps, cameras due to their advantages of large capacity and low associated costs, long cyclability life, and high energy density with no memory [3, 4]. The efficiency of lithiumion battery application requires an effective battery management system (BMS) with functional requirements of maintaining, monitoring, and estimating an accurate and precise state of charge (SOC) of the battery individually 
or in a stack $[5,6]$. However, LIBs require special handling to avoid deterioration of battery performance and prevent situations that could result in severe damage or explosions and reliability of the cell under operation [7]. The battery state of charge (SOC) is one of the most important states of a battery to keep track of to optimize performance [8]. The monitoring and regulation of the states of batteries are done by a BMS that uses a battery analytical model and consists of hardware and software for battery management and to ensure the safe operation of the battery and prolong life [9, 10]. The BMS includes, among others, algorithms to determine the battery's more critical states. For EV, electric motorcycles, balance cards, scooters, and smart grids applications, batteries must not only deliver a certain amount of energy to the drive train during operation but also provide power in different road situations [6]. For this reason, it is essential to know the maximum power that can be delivered to and from the battery by charging or discharging, respectively, with SOC being needed for making decisions on the operation.

The electrochemical model, neural network model, and the equivalent circuit model are three commonly used equivalent models of lithium-ion batteries, among which the equivalent circuit model has been widely used due to its simple structure, suitable accuracy, and the estimation of SOC of the battery by processing a large number of real-time input and output data of the battery $[11,12]$. To explore the performance differences between different model structures, [13] studied 12 kinds of equivalent circuit models with different structures, comprehensively analyzed the complexity and accuracy of the models, and concluded that the lower-order Resistor-Capacitor (RC) equivalent circuit models have better performance. Mastering the time-varying characteristics of model parameters is another key problem in the equivalent modeling of lithium-ion batteries [14, 15]. Factors such as battery aging, ambient temperature, and the charge-discharge rate will all have varying degrees of impact on model parameters $[16,17]$. Through a large number of experiments, exploring the functional relationship between model parameters and environmental factors, and then constructing corresponding model parameter compensation strategies, is one of the most commonly used methods to improve model accuracy. [15, 18, 19] Analyzed and established the relationship between the open-circuit voltage, state of charge, charging/discharging characteristics, and battery capacity, and corrected the model's open-circuit voltage by real-time estimation of battery state of charge and capacity. Similarly, the internal resistance and resistor-capacitor parameters of the equivalent model can also be updated through the corresponding mapping relationship [20, 21]. However, the cell aging status is difficult to accurately obtain, especially when the equivalent model parameters are unknown [22]. Therefore, how to make full use of the aging law of the battery equivalent model parameters without accurately knowing the current aging state of the battery in [23] is the key to solving the above problems.

The battery operations can be modeled to find and monitor their state, mainly the SOC. In estimating SOC two different approaches are used that's definition-based and model-based [24]. A large number of solutions have been proposed by researchers, which have been gradually applied to the SOC estimation process of lithium-ion batteries. A SOC estimation study was conducted based on the OCV (Open Circuit Voltage) method in [25]. The online SOC estimation was realized using the lagging OCV model in [25-27]. An overview was conducted for the SOC estimation methods of lithium-ion batteries in [28]. The advent of Electric Vehicles (EVs) and Hybrid Electric Vehicles (HEVs) is to reduce energy consumption and environmental pollution caused by general vehicles [29]. The main advanced power battery source in an electric vehicle and electronic devices are based on Nickel Metal Hydride battery (Ni-MH) and lithium-ion battery [30, 31]. A lithium-ion battery and Nickel Metal Hydride battery $(\mathrm{Ni}-\mathrm{MH})$ is characterized by high capacity, long life, and high-power density, the present to be the main energy storage system of the electric vehicle going with the declining costs [32]. The economy is another very important aspect that will determine whether they can survive in the market competition. Meanwhile, the real-time and accurate estimation of the state of charge (SOC) of the battery would have an impact on the economy of the hybrid electric vehicle $[33,34]$. Notwithstanding, considering the significant importance and wide range of applications, many challenges remain incapable in applications and cognizance to characterization and battery managements for lithium-ion battery technology [35]. To accommodate the optimal operating conditions for a battery, one should observe BMS facilitates safe and efficient charging/discharging of the battery [19]. The work of BMS also needs to control the battery charging and its discharging process to avoid a situation such as over-charging and overdischarging, which leads to premature wear-out of the designed operating voltages. The battery becomes unstable and may pose a safety hazard if over-charged [36, 37] stressing the battery and this may lead to damage and reduction in its lifespan [38]. The estimation of SOC generally requires modeling of the battery cell and packs, the 
structure of the battery model, and parameter identification should be based on characteristics of the chargedischarge test under different temperatures, which need sufficient test data of batteries. Equivalent circuit models commonly used for lithium-ion batteries are the Thevenin and RC models [39, 40].

To realize remaining battery life prediction, real-time battery management, and extending its useful life, more accurate monitoring of the battery's available energy and state of charge (SOC) is required. Two methods of monitoring the state of charge of lithium batteries are analyzed in this paper. Firstly, the OCV-SOC test method was used on the battery and the results were analyzed. The results were used to draw the OCV-SOC curve. The relationship between the OCV and SOC was determined through curve fitting in MATLAB. The results were compared with the results from the HPPC test. The HPPC test was also done with the same Lithium-ion battery and the data was analyzed with the OCV-SOC test results. Aiming at the goal of accurately describing the working state of Ternary lithium-ion battery, considering the accuracy and computational of the characterization, the Reduced-order UKF method is used to estimate the SOC of the lithium battery, which reduced the computational complexity of the traditional method. The rest of the paper is constructed as follows: The mathematical and theoretical analysis is conducted in section 2, including Thevenin modeling, state-space iteration, open-circuit voltage test, and the proposed HPPC-ROUKF algorithm for SOC estimation. In section 3, the experiments are illustrated as well as their estimation effect results. Conclusions and future works are finally reported in section 4.

\section{Mathematical Description}

\subsection{Thevenin RC modeling}

The model chosen here is the Thevenin RC modeling [41], which is simple yet accurate enough for the controloriented purpose in EVs. The branch loop of the circuit has $R_{P}$ and $C_{P}$, which are utilized to model the battery polarization effect. $R_{0}$ denotes the ohmic resistance and is responsible for the instantaneous voltage drop of the step response. $U_{O C}$ denotes the open-circuit voltage of the lithium-ion battery and it changes with different capacity levels. $U_{L}$ is the terminal voltage of the lithium-ion battery pack and $I_{L}(\mathrm{t})$ is the current value at both ends. $I_{L}$ denotes the terminal current (current flowing into the battery is considered positive).

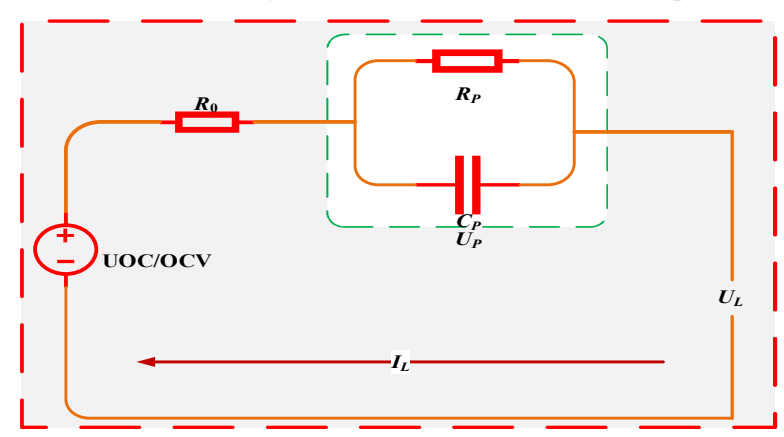

Figure 1. Thevenin RC modeling

In Figure 1, the method was adopted because the topology generally proposes a tradeoff between battery cell computational requirements and the approximation of the precision. That can be expressed in Equation (1).

$$
U_{L}=U_{O C}-U_{0}-U_{P}
$$

The model chosen here is the Thevenin RC modeling, based on Kirchhoff's law, the electrical behavior of the circuit can be characterized as a complete cycle of an HPPC test with pulse and voltage reading at very important periods. These voltages read from the point of discharging after resting $U_{1}$, then the point where the current was 70A while discharging $U_{2}$, followed by the endpoint of discharge $U_{3}$. The point of the sharp rise of voltage is recorded as $U_{4}$ and $U_{5}$ was the last point before charging. In the experiment, this cycle was repeated 9 times. After the test, some data were extracted and used for identifying ECM parameters like $R_{0}, R_{P}$, and $C_{P}$ 


\subsection{State-space iteration}

The mathematical description represents the state-space of a physical system as a set of state variables, input, and output related, to differential equations. According to Kirchhoff laws, the Thevenin RC modeling is analyzed, and the voltage and current expressions of the equivalent circuit are obtained as shown in Equations (2) and (3).

$$
\begin{aligned}
& \left\{\begin{array}{l}
U_{P}=\frac{R_{P}}{C_{P}}+\frac{I_{L}}{C_{P}} \\
U_{L}=U_{O C}(S O C)-U_{P}-I_{L} R_{0}
\end{array}\right. \\
& \left\{\begin{array}{l}
U_{L}=U_{O C}-U_{0}-U_{P} \\
I_{L}=C_{P} \frac{d U_{P}}{d t}+\frac{U_{P}}{R_{P}}
\end{array}\right.
\end{aligned}
$$

Among them, the open-circuit voltage can be characterized by the state variable state of charge (SOC), and a nonlinear function relationship can be obtained. Using the knowledge of modern control theory, the equivalent circuit model is discretized. The definition of SOC is shown in Equations (4) and (5).

$$
\begin{gathered}
\left\{\begin{array}{l}
I_{L}=\frac{U_{P}}{R_{P}}+C_{P}=\frac{U_{P}}{d t} \\
U_{L}=U_{O C}(S O C)-U_{P}-I_{L} R_{0}
\end{array}\right. \\
U_{L}=U_{O C}(S O C(k))-U_{P}(k)-I_{L} R_{0}(k)
\end{gathered}
$$

Where $R_{P}$ and $C_{P}$ represent the polarization resistance and polarization capacitance respectively. The estimate of the time and the observation of the current time update the estimate of the state variable. The Kalman filter algorithm estimates the essence of the lithium-ion battery SOC by using the ampere-hour (Ah) integration method to calculate the SOC and uses the measured voltage value to correct the SOC value obtained by the ampere-hour integration method equation.

After taking the reading of the OCV with its corresponding SOC, the values were input into MATLAB and its curve-fitting tool was used to determine the best relationship between them. Open Circuit Voltage-Based Estimation (OCV) SOC estimation methods commonly impose a characterization of the OCV curve (mainly through a polynomial or a look-up table), as they use either a direct OCV curve inversion method (if the application permits cell steady-state voltage measurement), or a cell model-based method. Making voltage measurements to determine the SOC for the cell allows us to define the relation. Open-circuit voltage (OCV) refers to the potential difference between positive and negative electrodes when the external circuit is disconnected. After a certain period of shelving, the terminal voltage of the battery is approximately equal to the open-circuit voltage. There is a relatively fixed functional relationship between the OCV and SOC, as shown in Figure 2(a).

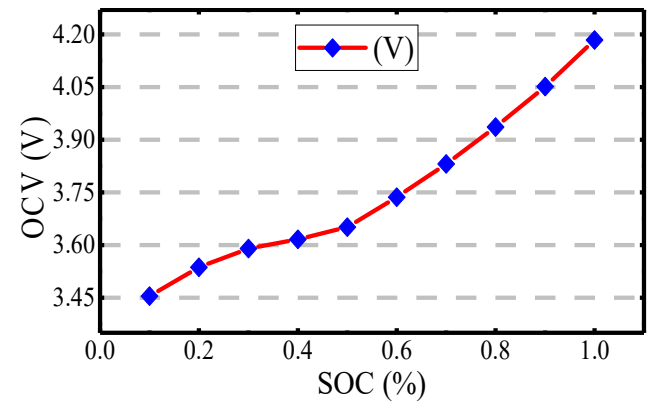

(a): Relations between SOC and OCV

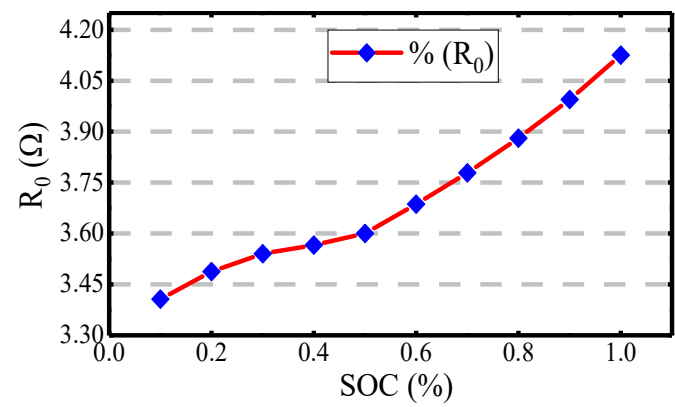

(b): Relations between SOC and $R_{0}$ 


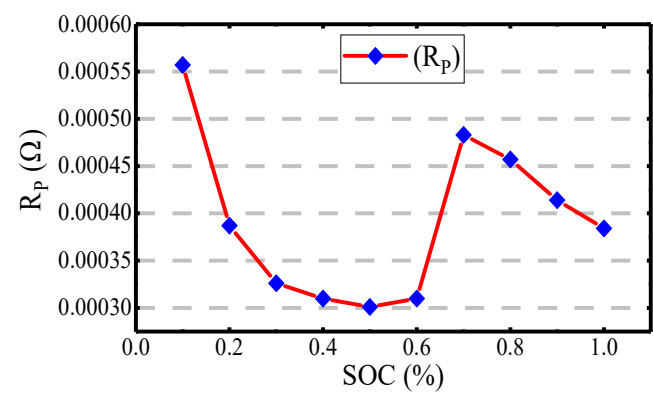

(c): Relations between SOC and $R_{P}$

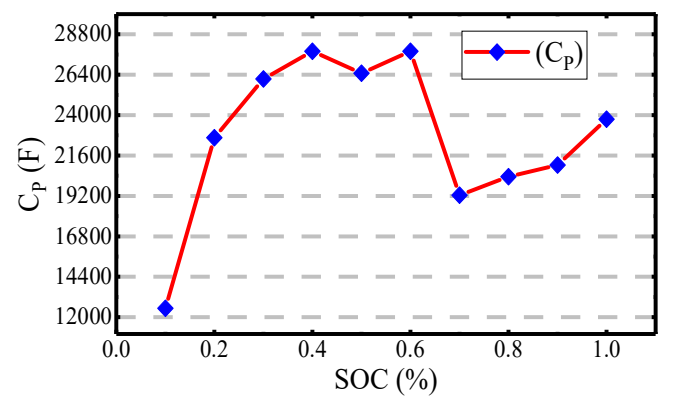

(d): Relations between SOC and $C_{P}$

Figure 2. Relationship between SOC and OCV with $R_{0}, R_{P}$, and $C_{P}$

The OCV of the battery at each SOC point is measured, and then the OCV-SOC curve, which changes with SOC, is drawn. The corresponding functional relationship between OCV and SOC can be obtained by curve fitting tools in MATLAB as shown in Equation (6)Error! Reference source not found. .

$$
S O C=f^{-1}(O C V)
$$

The relationship between OCV and SOC of the LIB can be observed in Figure 2(a). It was found that OCV and SOC have nonlinear positive correlations and from the curve in the above figure, the OCV decreases as the SOC decreases until SOC was 0 at $4.20 \mathrm{~V}$. The discrete points of the OCV-SOC relationship of the LIB were obtained through the intermittent discharge process of the test and the overall variation curve was achieved by using the curve fitting method. The result of the fitting function is given by equation $\left\{\mathrm{a}-\mathrm{b}^{*}(1-\exp (-\mathrm{x} / \mathrm{c}))\right\}$.

In Figure 2(b), $R_{0}$, representing the battery internal "ohmic" resistance was plotted against the SOC. In the above figure, the ohmic resistance was high at the initial stage but dropped sharply because the battery was losing its energy. The derived equation for the ohmic internal resistance calculation is as shown in Equation (7).

$$
R_{0}=\frac{\left(U_{1}-U_{2}\right)+\left(U_{4}-U_{3}\right)}{2 I}
$$

From Figure 2(c), $R_{P}$, representing the battery internal polarization resistance was also plotted against SOC to determine its relationship. It was observed that $R_{P}$ was fluctuating until at $0.6 \mathrm{SOC}$ where there was a sharp increase from 1 to 7 . The steep increase was due to the loss of voltage in the battery. The equation that best describes the relation is the corresponding weights as shown in Equation (8).

$$
R_{P}=\frac{U_{1}-U_{3}-R_{0} I}{I \frac{t_{2}-t_{1}}{(1-e \tau)}}
$$

In Figure 2(d), the relationship between the $C_{P}$ - Shunt capacitance (around $R_{P}$ ) was identified. The $C_{P}$ values were plotted against SOC values by adjusting the degree to get a suitable shape. From the graph, the $C_{P}$ drops and ascends sharply and was a little flat from SOC 0.8 to 0.7 but dropped again. At 0.4 SOC, its capacitance dropped off and couldn't rise again till the end of the test. The relationship between the $C_{P}$ and SOC is non-linear. The derived equation for the relationship between the $C_{P}$ and SOC was determined and the value of capacitor $C_{P}$ can be directly obtained in Equation (9).

$$
C_{P}=\frac{t_{4}-t_{3}}{R_{P} \operatorname{In}\left(1 \frac{U_{4}-U_{3}}{U_{1}-U_{3}}\right)}
$$

The fitted parameter is then shown with a polynomial of the 6 th order, and the following results are obtained. The fitting results of the curve are $R_{0}, R_{P}$, and $C_{P}$, respectively, reflect the parameters for the equivalent circuit of the model. The fitted results are shown in Equation (10(10). 


$$
\left\{\begin{array}{l}
\left(U_{O C}\right)=20.08 * x^{6}-61.22 * x^{5}+68.12 * x^{4}-32.07 * x^{3} \\
+5.234 * x^{2}+0.6794 * x+3.36 \\
\left(R_{0}\right)=0.009028 * x^{6}-0.02961 * x^{5}+0.03699 * x^{4}-6306 * x^{3} \\
+0.006988 * x^{2}-0.001097 * x+0.001336 \\
\left(R_{P}\right)=0.1186 * x^{6}-0.4265 * x^{5}+0.5976 * x^{4}-0.4095 * x^{3} \\
+0.1413 * x^{2}-0.02288 * x+0.001786 \\
\left(C_{P}\right)=-4.098 e+06 * x^{6}+1.527 e+07 * x^{5}-2.213 e+07 * x^{4} \\
+1.566 e+07 * x^{3}-5.6 e+06 * x^{2}+9.558 e+05 * x-4.063 e+04
\end{array}\right.
$$

The open-circuit voltage is calculated as shown in Equation (11):.

$$
U_{O C}=U_{1}-U_{P}=U_{1}\left(1-e^{t / \tau}\right)
$$

The time constant is calculated as shown in Equation (12):.

$$
\tau=-\frac{t_{4}-t_{3}}{\operatorname{In}\left(1 \frac{U_{4}-U_{3}}{U_{1}-U_{3}}\right)}
$$

Based on the Thevenin RC modeling of the battery and its state space description, the specificity of the SOC estimation under the power group application condition was analyzed. The adaptive SOC estimation model framework for the combined conditions is shown below in Error! Reference source not found.

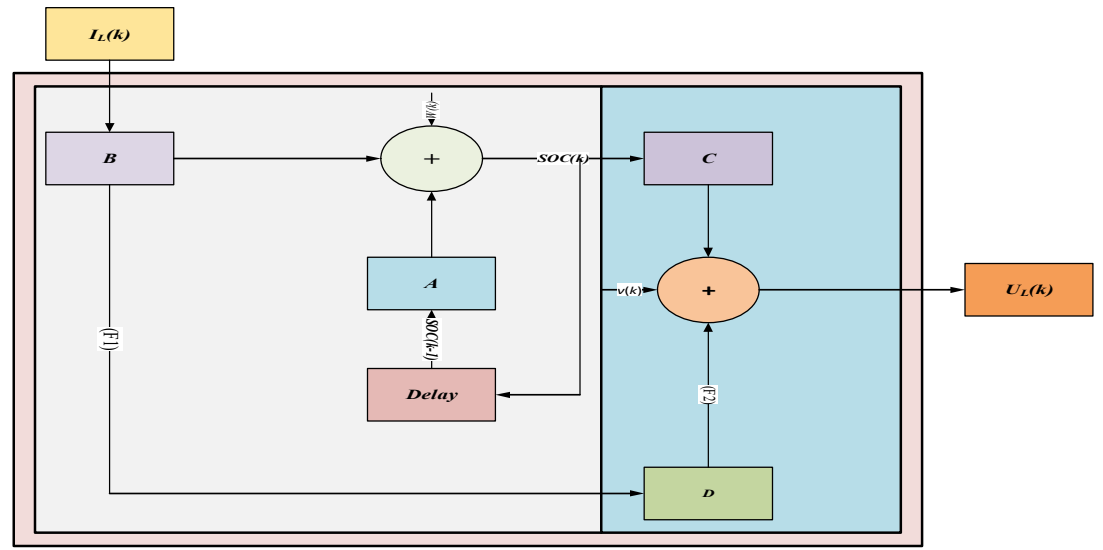

Figure 3. The SOC estimation model framework

In Error! Reference source not found., the $I_{L}(k)$ represents the current input by the system build, and $U_{L}(k)$ is the closed-circuit loop voltage output by the system build. The $\mathrm{SOC}(k)$ is the SOC value at $k$ time point, and $\operatorname{SOC}(k$ $1)$ is the SOC value at $k-1$ th-time point. The whole graph can be represented by the discrete space description of the nonlinear SOC estimation model. For (F1) it represents the state equation and (F2) represents the observation equation, and then the iterative calculation is realized by combining the discrete processing. By iteratively using the expression $\mathrm{SOC}=\mathrm{SOC}(k-1)$ and replacing the effective parameter with the $\mathrm{SOC}$ value, an equation that is closely related to time can be obtained and used for the subsequent parameter replacement and characterization. Under the influence of random noise, the estimation process at different time points can be obtained.

$$
\left\{\begin{array}{l}
S O C(k \mid k-1)=S O C(k-1)-\frac{\eta_{I} \eta_{\tau} I_{L}(k) T_{s}}{Q_{n}} K_{s} T_{s} \\
\left.U_{L}(k)=\left(U_{O C}-U_{\delta}\right)-R_{0}-R_{\delta}\right) I_{L}(k)-I_{L}(k) R_{P}\left(1-e^{-T_{s} /\left(R_{P} C_{P}\right)}\right)-I_{L}(k) R_{c d}
\end{array}\right.
$$


Based on the equivalent model of the battery and its state-space description, the specificity of the SOC estimation under the power group application condition is analyzed. The adaptive SOC estimation model framework for the combined conditions is shown in Equation (14).

$$
\operatorname{SOC}^{(i)}(k-1)=\left[\begin{array}{l}
\operatorname{SOC}(k-1) \\
\operatorname{SOC}(k-1)+\sqrt{(n+\lambda) P(k-1)} \\
\operatorname{SOC}(k-1)+\sqrt{(n+\lambda) P(k-1)}
\end{array}\right]
$$

The first-order prediction of the sinusoidal data point sequence should be calculated with the length of $2 n+1$ as shown in Equation (15).

$$
\operatorname{SOC}^{(i)}(k-1)=f\left[k, S^{(i)}(k-1)\right], i=1,2, L, 2 n+1
$$

Where $\mathrm{n}$ is the dimension of the state variable, $\lambda$ is the scaling parameter, SOC is the mean of the state variable, and $P$ is the covariance matrix. The corresponding weights are shown in Equation (16).

$$
\operatorname{SOC}^{(i)}(k \mid k-1)=\left[\begin{array}{l}
\operatorname{SOC}(k \mid k-1) \\
\operatorname{SOC}(k \mid k-1)+\sqrt{(\eta+\lambda) \mathrm{P}(k \mid k-1)} \\
\operatorname{SOC}(k \mid k-1)+\sqrt{(n+\lambda) \mathrm{P}(k \mid k-1)}
\end{array}\right]
$$

The status update is calculated as shown in Equation (17).

$$
\operatorname{SOC}(k)=\operatorname{SOC}(k \mid k-1)+\mathrm{K}(k)\left[U_{L}(k)-U_{L}(k \mid k-1)\right]
$$

This method implements the estimation process based on the Kalman framework in the estimation process but does not require the nonlinear equation linearization calculation as the traditional SOC estimation methods.

\subsection{Reduced-order UKF Estimation}

To illustrate the UKF of the state estimation, a new application example corresponding to noisy time series estimation is provided. Combined with Thevenin RC modeling, considering the practical application, only SOC is selected as the system state variable, and the terminal voltage $U_{L}$ of the battery is taken as the observed variable of the system. The established battery state-space expression is as shown in Equation (18).

$$
\left\{\begin{array}{l}
S O C_{k+1}=S O C_{k}-I_{L k} \frac{\Delta t}{Q_{N}}+w_{k} \\
U_{L, k+1}=f\left(S O C_{k+1}\right)-U_{0}-U_{P}-v_{k+1}
\end{array}\right.
$$

In the formula, the SOC value at time $k+1$ is predicted at time $k, \Delta t$ is the sampling interval time, $Q_{N}$ is the rated capacity of the battery, and the actual value is subjected to capacity calibration. $I_{L k}$ is the current at time $k$, and the charging direction is positive. The output variable $U_{L, k}$ is selected. $U_{L, k+1}=f\left(S O C_{k+1}\right)$ is a relationship between the open-circuit voltage and the SOC of the lithium battery? $w_{k}$ and $v_{k+1}$ are process noise and observed noise, respectively.

For the nonlinear problem of lithium-ion batteries, UKF does not adopt the way of forced linearization of the system but obtains a certain number of sampling points near the state estimation according to the sampling theorem and the statistical principle [42]. The sampling point is usually called the Sigma point and the condition that the Sigma point needs to satisfy is that it has the same mean value and covariance as the original state to ensure the transitivity to the original state. Then, the Sigma point set is nonlinearly transmitted using the state equation, the transformed mean and the optimal solution are obtained in the iterative process. This approximation is essentially a statistical approximation rather than a solution but has a higher computational accuracy for the statistics of nonlinear distributions. The implementation of reduced-order UKF can be divided into two stages: prediction and update. According to the symmetric sampling strategy, the Sigma point is selected as the sampling point and needs to satisfy the same mean and covariance as the original state. Let the value of the $S O C_{k}$ state variable at the time $k$ known and the error variance matrix $P_{k}$. The implementation principle and the specific process is as follows. 


\section{Prediction stage}

The estimated value of the system state variable at time $k+1$, as shown in Equations (19).

$$
\operatorname{SOC}^{(i)}(k+1 \mid k)=f\left[\operatorname{SOC}^{i}(k), u_{k}\right]
$$

The algorithm uses the last time point instead of the SOC in the state space function, and only one calculation is needed to obtain the predicted SOC value. The predicted SOC was obtained by using Equation 17. The predicted value of the SOC state variance can be obtained as shown in Equation (20).

$$
\operatorname{SOC}(k \mid k-1)=\sum_{i=0}^{2 n} \omega^{(i)} S O C^{(i)}(k \mid k-1)
$$

Where, $S O C^{i}(k)$ is the sampling point obtained by using the unscented transformation, $i=1,2,3, \ldots, 2 n+1, u_{k}$ is the input variable. The sampling point is predicted in one step by combining Equation (20) with ......, and the mean value of the system state quantity prediction is obtained by weighted summation as shown in Equation

(20). Similarly, the error variance matrix of $k+1$ time is predicted as shown in Equation (21).

$$
\mathrm{P}(k \mid k+1)=\sum_{i=0}^{2 n} \omega^{(i)}[S O C(k \mid k+1)-(k \mid k+1)]\left[S O C^{(i)}(k \mid k+1)\right]^{T}+Q_{k+1}
$$

The Sigma sampling points are updated and $\operatorname{SOC}(k \mid k+1)$ is obtained from Equations (20) and (21), and the sampling points are updated by combining Equation (6). Substituting the state quantity predicted value point set at time $k+1$ into the observation equation, using the observation equation to obtain the predicted value of the $k+1$ time observation, and performing a state transfer in the observation equation. Then, the weighted sum is calculated to obtain the mean value of the measurement amount at time $k+1$, as is shown in Equations (22) and (23).

$$
\begin{gathered}
U_{L}^{i}(k+1 \mid k)=h\left[\operatorname{SOC}^{i}(k+1 \mid k), u_{k}\right] \\
U_{L}(k+1 \mid k)=\sum_{i=0}^{2 n} \omega_{m}^{i} U_{L}(k+1 \mid k)
\end{gathered}
$$

The variance matrix of the measured values, the covariance of the state quantity, and the measured quantity at time $k+1$ is calculated as shown in Equation (24).

$$
\left\{\begin{array}{l}
P_{U_{L}(k+1) U_{L}(k+1)}(k+1 \mid k)=\sum_{i=0}^{2 n} \omega_{c}^{i}\left[U_{L}(k+1 \mid k)-\stackrel{\lambda}{U}_{L}^{\lambda}(k+1 \mid k)\right]\left[U_{L}^{i}(k+1 \mid k)-\stackrel{\lambda}{U}_{L}^{\lambda}(k+1 \mid k)\right]^{T}+R_{k+1} \\
P_{S O C(k+1) U_{L}(k+1)}=\sum_{i=0}^{2 n} \omega_{c}^{i}\left[\operatorname{SOC}^{i}(k+1 \mid k)-S O C(k+1 \mid k)\right]\left[U_{L}^{i}(k+1 \mid k)-\stackrel{\lambda}{U}_{L}(k+1 \mid k)\right]^{T}
\end{array}\right.
$$

\subsubsection{Update state-space equation}

2) Update phase

The Kalman gain, update system state variable values, and the error variance matrix are calculated, as shown in Equation (25).

$$
\left\{\begin{array}{l}
K_{k+1}=P_{U_{L}(k+1) U_{L}(k+1)} / P_{S O C(k+1) U_{0}(k+1)} \\
\operatorname{SOC}(k)=S O C(k+1 \mid k)+K_{k+1}\left[U_{L}(k+1)-U_{L}(k+1 \mid k)\right] \\
P(k+1)=P(k+1 \mid k)-K_{k+1} P_{U_{L}(k+1) U_{L}(k+1)} K_{k+1}^{T}
\end{array}\right.
$$

The Reduced-order UKF method implementation process is shown in Figure 4. Here, since the state variable is only one dimension, the number of selected Sigma points is 3. The Sigma point is passed through the system state equation to derive the predicted point group. Then, the Kalman gain and the error between the true value of the 
observed variable and the prediction are used to continuously correct the predicted value. The correction principle is the same as KF, that is, the correction is large when the deviation is large, and the correction range is small when the deviation is small. Finally, the optimal estimate of the system state variables can be obtained.

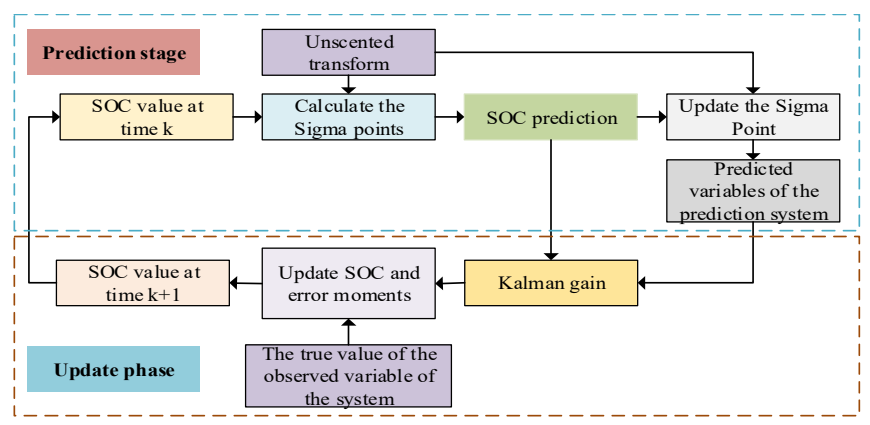

Figure 4. ROUKF Implementation process diagram

As shown in Figure 4, the parameters and weights determined by the unscented transformation are prepared for the state quantity Sigma points. In the Sigma points finding module, the Sigma points with the same mean and covariance of the original state are obtained according to the symmetric sampling strategy. The obtained Sigma points perform a nonlinear transfer in the state prediction module, and the weighted summation obtains the mean value of the state quantity predicted value at the next time. The sigma points are then updated by the predicted mean and the UT module. The updated Sigma points predict the observation value according to the observation equation, obtain the Kalman gain, and the correction parameters. In the state variable and error matrix updates module, the predicted value means and the corresponding error covariance are updated according to the real-time observation data and the Kalman gain until the next iteration is completed.

\section{Experimental Verification Analysis}

\subsection{Implementation of the test platform}

Mastering the basic working characteristics of the lithium-ion battery, including the open-circuit voltage characteristics, temperature, rate, and aging characteristics is an important basis for battery equivalent modeling and state of charge (SOC) estimation. The accuracy of battery open-circuit voltage has a significant effect on SOC estimation performance. Therefore, this section focuses on the analysis of the open-circuit voltage characteristics of a battery. To obtain the basic characteristics of battery charge and discharge, the following experimental platform was built as shown in Figure 5.

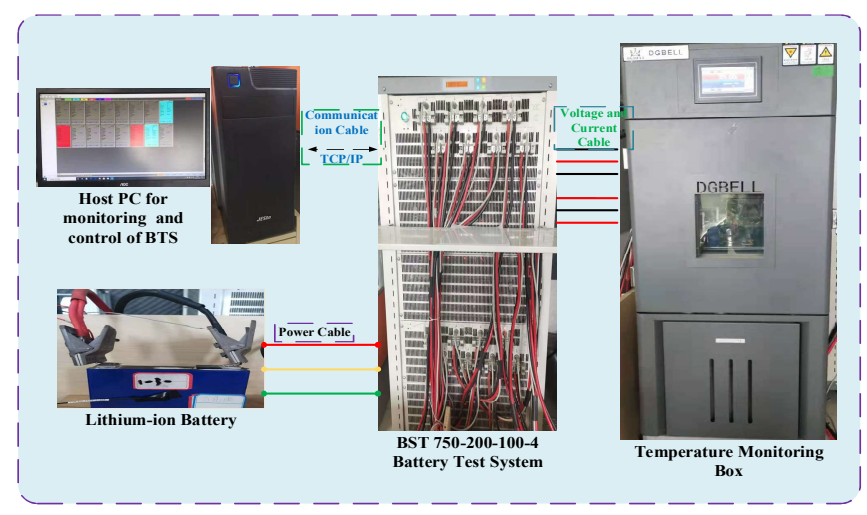

Figure 5. The sketch of the battery testing platform

Figure 5, shows the lithium-ion battery test platform built, mainly including the BTS 750-200-100-4 battery test equipment, the incubator, 70Ah power lithium-ion battery, and a home computer. The black line in this figure indicates TCP/IP data transmission connection, and the red line is an electrical connection. Based on the above experimental platform, the following experiments were carried out. 
Table 1. Basic technical parameters of the battery

\begin{tabular}{|l|l|}
\hline Factor & Parameter \\
\hline Cell Nominal Capacity/Ah & 70 \\
\hline Size: Length * Width * Height /Mm & $14 * 38^{*} 91$ \\
\hline Rated Nominal Voltage/V & 3.7 \\
\hline Charge Cut-Off Voltage/V & $3.0 \mathrm{C}$ \\
\hline Discharge Cutoff Voltage/V & $4.2 \pm 0.05$ \\
\hline Working Temperature $/{ }^{\circ} \mathbf{C}$ & 2.4 \\
\hline Rated Capacity/Ah & $-15 \sim 70$ \\
\hline
\end{tabular}

\subsection{Determination of Parameters}

The comparison of the two methods of SOC estimation was made out of experiments conducted and some references made from other papers and articles. In the experiments, the same lithium-ion battery was used for both methods. The process of each method is described and the results analyzed. The Hybrid Pulse Power Characterization (HPPC) test is intended to determine the dynamic power capability over the device's usable voltage range using a test profile that incorporates both discharge and region pulses. Data from the HPPC test includes the voltage response curve, response time constants during discharge, rest, and region operating regimes. This data is used to evaluate resistance degradation during subsequent life testing and to develop hybrid battery performance models for vehicle systems analysis. HPPC tests provide the necessary data to quantify the battery capacity and estimate dynamic characteristics. The process for the test is as follows;

1. The battery is fully charged

2. The battery is left to rest for $30 \mathrm{mins}$

3. The battery is discharged for 10 seconds

4. The battery is left to rest for 40 minutes

5. A 10 -second charge pulse is performed

6. The battery is left to rest for 30 minutes

7. Cycle continues from 3 to 6 for 9 other times and ends

Table 2, shows the result from the HPPC test matching the SOC to each OCV and the battery parameters that were obtained after computations.

Table 2. Model parameters under different SOC

\begin{tabular}{|c|c|c|c|c|c|}
\hline SOC & UOC/OCV & $“ R_{0}(\Omega) "$ & $“ R_{P}(\Omega) "$ & “C $C_{P}(F) "$ & $I(A)$ \\
\hline $100 \%$ & 4.1840 & 4.12554 & 0.000384 & 23758.36 & 70 \\
\hline $90 \%$ & 4.0513 & 3.994727 & 0.000414 & 21033.79 & 70 \\
\hline $80 \%$ & 3.9360 & 3.881074 & 0.000457 & 20340.74 & 70 \\
\hline $70 \%$ & 3.8309 & 3.778826 & 0.000483 & 19245.86 & 70 \\
\hline $60 \%$ & 3.7366 & 3.686263 & 0.00031 & 27796.68 & 70 \\
\hline $50 \%$ & 3.6511 & 3.600253 & 0.000301 & 26486.94 & 70 \\
\hline $40 \%$ & 3.6163 & 3.565963 & 0.00031 & 27796.68 & 70 \\
\hline $30 \%$ & 3.5900 & 3.540066 & 0.000326 & 26145.8 & 70 \\
\hline $20 \%$ & 3.5367 & 3.487571 & 0.000387 & 22662.86 & 70 \\
\hline $10 \%$ & 3.4545 & 3.406629 & 0.000557 & 12523.86 & 70 \\
\hline
\end{tabular}


Figure 6 , shows the overall process of the test at a starting voltage of 4.2 volts and a cut-off voltage of 2.4 volts. The cut-off voltage was set to 2.4 volts to protect the battery from reaching its minimum voltage. The change rule of each parameter is obtained by the HPPC experiment, in which the current change is set as shown in Figure 6(b). Furthermore, the experimental results can be obtained by the HPPC experiment, in which the voltage change is shown below in Figure 6(d).

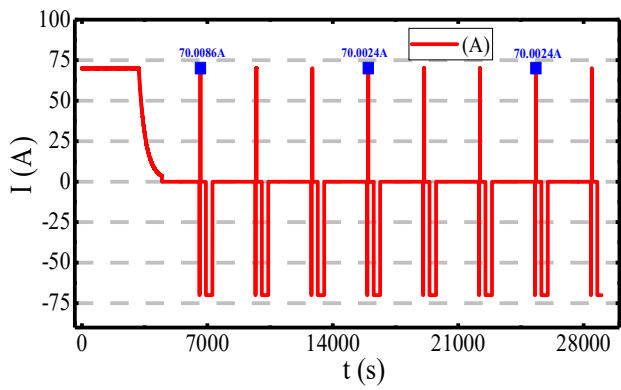

(a): HPPC test current of the main change

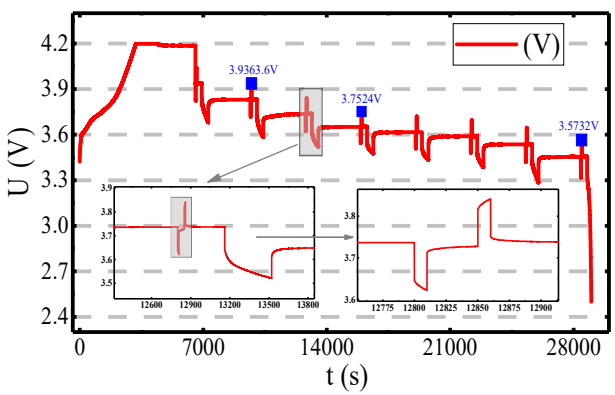

(c): HPPC test experimental voltage response

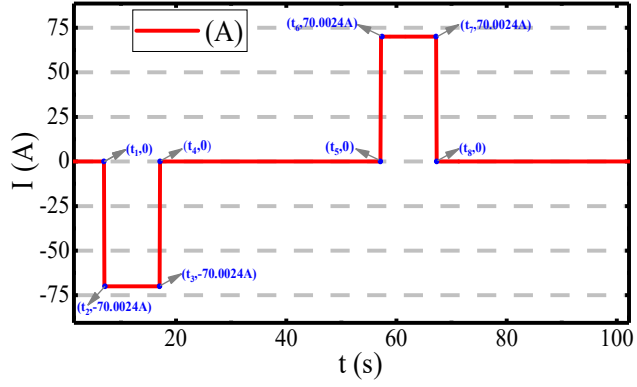

(b): Schematic diagram of loop current change

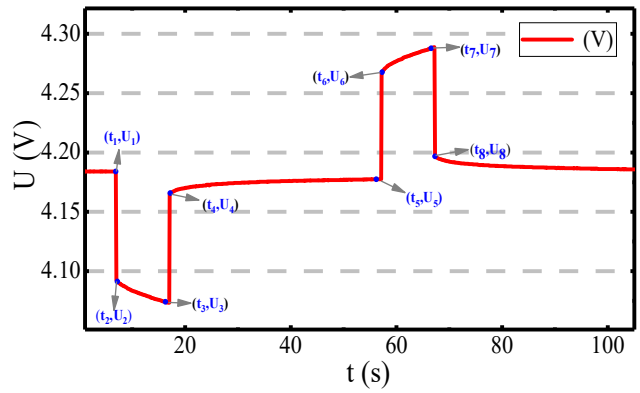

(d): Voltage diagram loop change

Figure 6. Pulse discharge test index curves

Figure 6(a), represents the complete current curve, and Figure 6(b) a single current curve Figure 6(c), represents the complete cycle of the HPPC test with the pulse and voltage reading at very important periods, and Figure 6(d) represents a single voltage curve. According to Figure 6(d), the voltage reading from the point of discharging after resting $U_{1}$, then the point where the current was 70A while discharging $U_{2}$, followed by the endpoint of discharge $U_{3}$. The point of the sharp rise of voltage is recorded as $U_{4}$ and $U_{5}$ was the last point before charging. In the experiment, this cycle was repeated 9 times. After the test, some data were extracted and used for identifying the ECM parameters like $R_{0}, R_{P}$, and $C_{P}$. The capacity experiment analysis was conducted for calibration of the capacity and energy of the battery. Constant current charging and discharging cycles are carried out and the capacity usually only takes into account the strain of material reactions. However, energy and power are different since the voltage component is still used. Figure 7(a) and (b), is the capacity and energy variation curve with time and it can be deduced from the figure that the capacity of the battery is approximately 70Ah. The maximum values obtained in the experiment are 69.053 Ah and 26676Wh. The higher the voltage platform, the higher the output and the change in time, and the changing direction of the two variables over time are approximately the same as seen in Figure 7. 


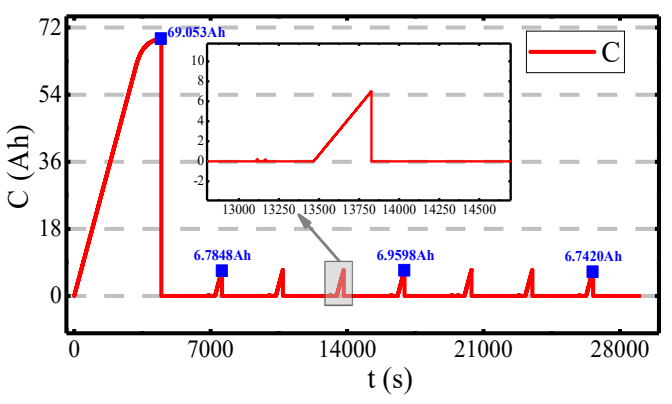

(a): Capacity diagram loop change

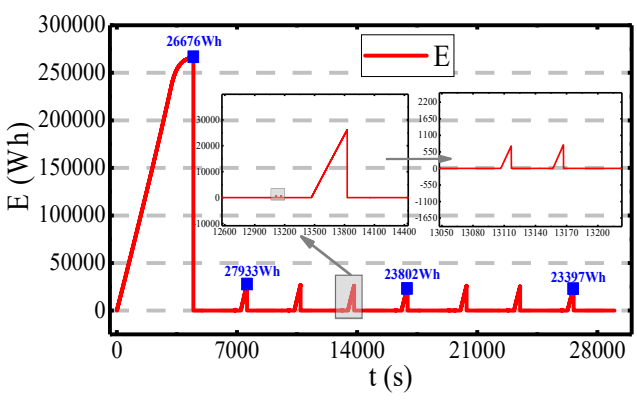

(b): Energy diagram loop change

Figure 7. Capacity and Energy transition waveform effect

Figure 7(a) depicts the capacity curve and Figure 7(b) represents the energy variation curve. The error waveform analysis reveals that the modified algorithm is adequate and the error is significantly decreased relative to the minimal. Since the given initial value varies significantly from the initial value, the battery's internal polarization effect is improved at the initial discharge point. The error in the state calculation of two algorithms at the beginning of the discharge is greater than at the later level.

\subsection{OCV-SOC Test Result}

The OCV method can be very accurate, but as it needs a rest time to estimate the SOC, it cannot be used in realtime. Comparing OCV-SOC at different temperatures gives different curves. The OCV-SOC characteristic curve is greatly influenced by the temperature change cannot be used for the estimation of SOC accurately, however, it can be used as a tool for estimating other methods. The OCV-SOC relationship is shown in Figure 8.

Table 3. Values from experiment SOC and OCV

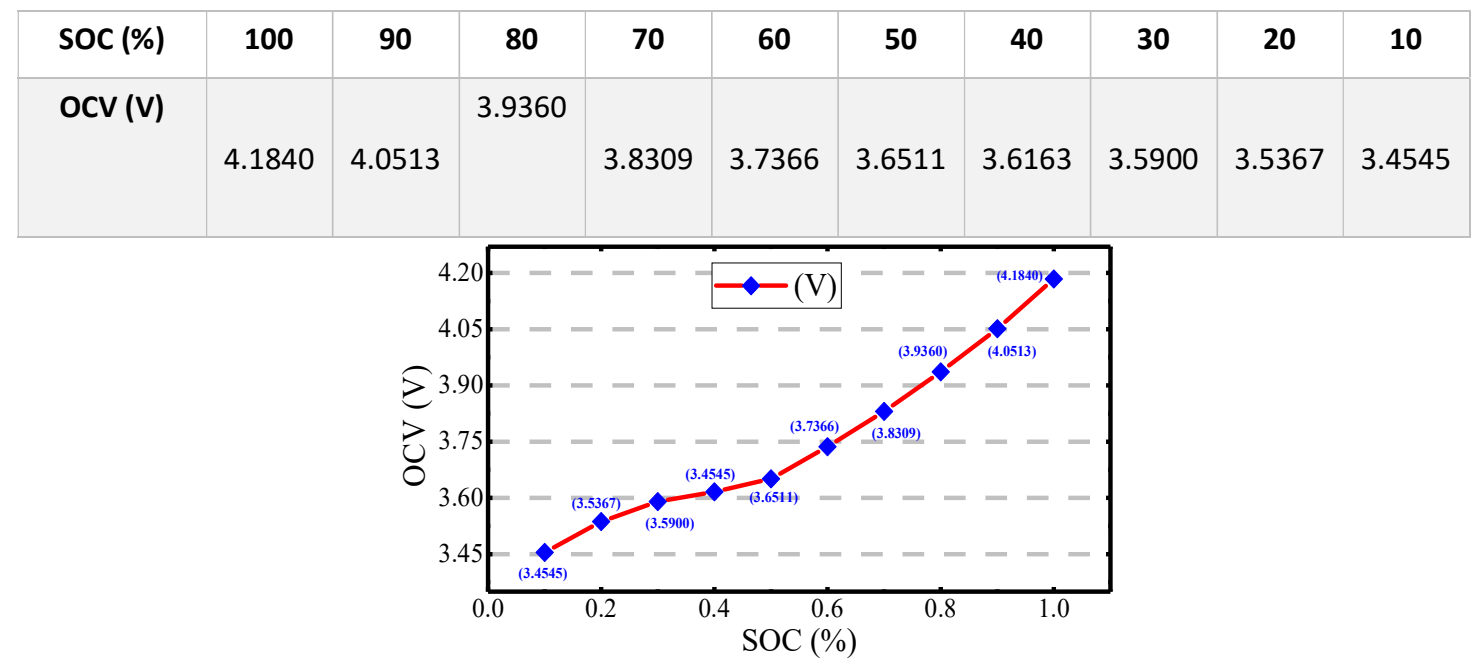

Figure 8. The relation between SOC-OCV curve

In the OCV method, the battery's voltage is continuously measured, and the corresponding SOC is obtained from the experiment. In the OCV-SOC test, the lithium-ion battery was charged to the full capacity of $4.2 \mathrm{~V}$ at $70 \mathrm{Ah}$. The battery was left to rest for about forty (40) minutes and slowly discharged for 10 seconds. After discharging, the battery is rested for another (30) minutes to step into another SOC level. The cycle continued until the SOC reached 0.1 after starting from 1 . The results are shown in the table above with the graphical representation of their relations.

\subsection{SOC estimation and Simulation Verification}

The values $R_{0}, R_{P}$, and $C_{P}$ were all substituted into the algorithm in a mean way and the established test battery model was a three-unit 70Ah lithium-ion battery of AVIC. Figure 9, shows the comparison of the voltage and 
current curves from the HPPC experiment and the variation with time. The overlaying curves shown in the figure depict the real or actual occurrence with the battery at specific times.

(a): Complete cycle voltage and current

curve (b): Single-cycle voltage and current curve

Figure 9. HPPC test cycles experiment effect

After identifying the parameters of the model build, the dynamic simulation model of the Thevenin RC modeling was constructed in Simulink. The identified parameters were put into the simulation model, the different working currents were input, the voltage response of the model was compared with actual voltage data, the model was verified, and validated the Simulink simulation structure, as shown in Figure 10(a). The built model validates the Simulink simulation structure to calculate SOC with discharge current with the application of the method of the ampere-hour (Ah) integral method, as shown in Figure 10(b). The internal structure of the Thevenin RC modeling is Figure 10(c), where each circuit component is a controllable parameter that changes over time. The model has five inputs and one output. The inputs are $I, R_{0}, R_{P}, C_{P}$, and $U_{O C}$ and the output, the terminal voltage $U$. This model can simulate the working condition of the lithium-ion battery. To evaluate the accuracy of the model parameters, the variable operating current is added to the model and the difference between the model output voltage and the time output voltage is compared. The Simulink model verification structural diagram is shown in Figure 10.

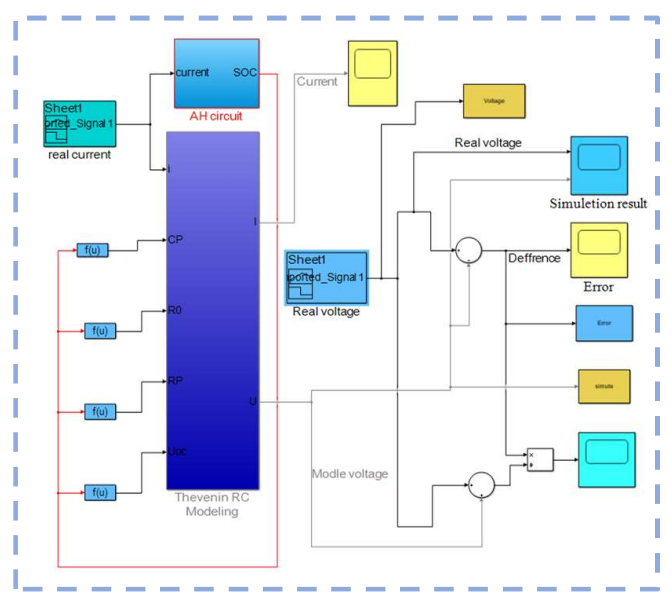

(a): Terminal voltage verification model

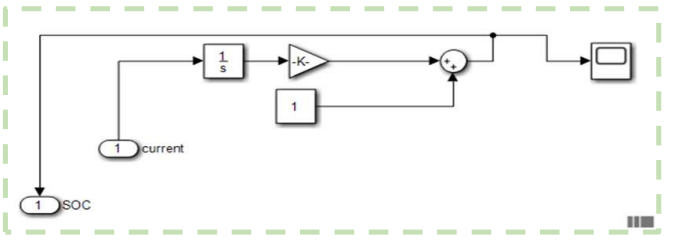

(b): Inner structure of amperehour (Ah)

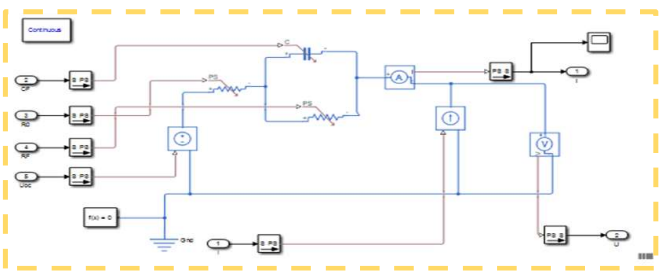

(c): Inside the Thevenin RC modeling in Simulink

Figure 10. Simulink Model Verification Structural Diagram

An accurate, intuitive, and comprehensive electrical model, which includes $I, R_{0}, R_{P}, C_{P}$, and $U_{O C}$, was built to capture the dynamic characteristics of a battery. The modeling accuracy will degrade when the operating conditions change because the model parameters are time-varying. The test battery model was a three-unit 70Ah lithium-ion battery of AVIC Lithium-ion Battery. The Thevenin RC modeling is established through parameterization with HPPC experimental results. To verify the validity of the SOC estimated values in the simulation, the results are compared with and the results from the HPPC experiment and the computations done thereafter. The value of current (I), in the experimental data obtained by the test equipment, is taken as the input condition, and the simulation terminal voltage is obtained through the simulation model and the experimental terminal voltages are compared to obtain the results as shown in Figure 11. 


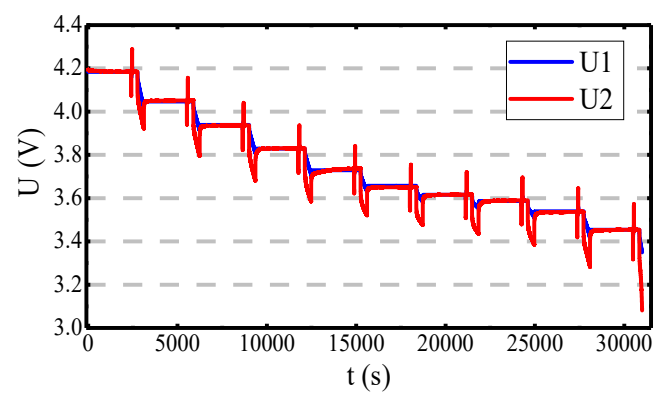

(a): Voltage comparison curve

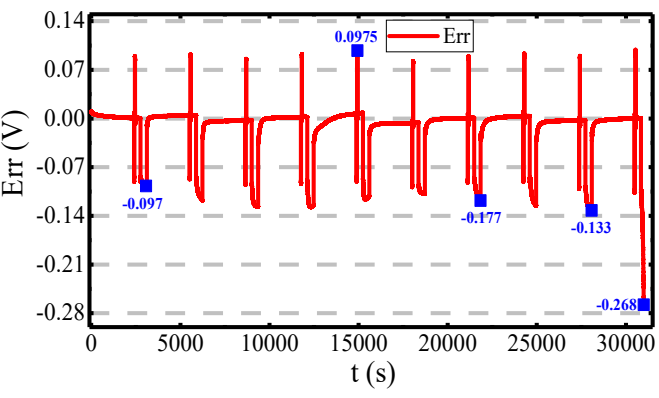

(b): Voltage estimation error curve

Figure 11. The model verification output result effect

In the experiment, the values of $R_{0}, R_{P}$, and $C_{P}$ were all substituted into the algorithm in a mean way. In Figure 11(a), and Figure 11(b) the variation among the original experiment results, calculated voltage, and simulated voltage is the modeling error. The simulated outcome in comparison to the original data is present in Figure 11(a), SOC explain in Equation (26).

$$
S O C=S O C_{0}-\int_{0}^{t} \frac{I_{L}(t)}{C_{n}} d t
$$

It can be seen from the figure that the predicted value curve gradually approaches the true value curve as the number of filtering increases, and fluctuates around the true value curve. The error value also gradually shrinks from the initial large error and eventually fluctuates around 0 , showing a tendency to converge.

\subsection{Simulation Verification Analysis}

To verify the estimation effect of the algorithm, the LIB test under Beijing Bus Dynamic Stress Test (BBDST) condition was performed on the ternary LIB shown in Figure 12.

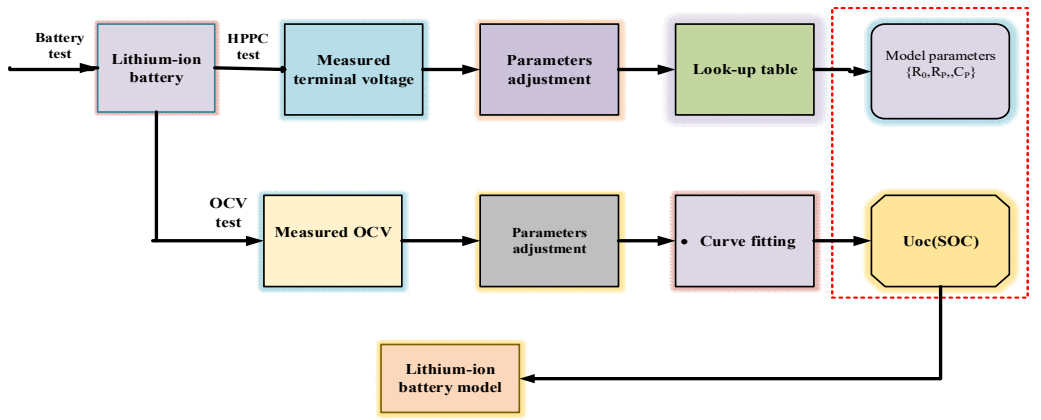

Figure 12. BBDST working condition parameters and description

The Beijing Bus Dynamic Stress Test (BBDST) working condition test used the lithium battery LFP70AH ternary lithium battery. The BTS750-200-100-4 battery testing equipment provided by Shenzhen Yakeyuan Technology Co., Ltd was used. The BBDST working condition is the actual data acquisition of the Beijing bus. Since the experiment was carried out on a battery of 70Ah, the data in $P c(w)$ was obtained by reducing $P h(k W)$, and $P c(w)$ was used for the experiment of the LFP70Ah ternary lithium battery. It can be seen from Table 2, that the time of a complete BBDST is $300 \mathrm{~s}$, the BBDST condition test is performed 20 times on the battery. The BBDST condition test data is shown in Figure 13. 


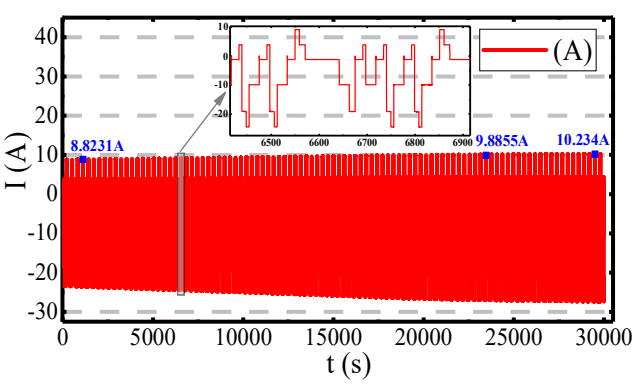

(a): BBDST working condition current

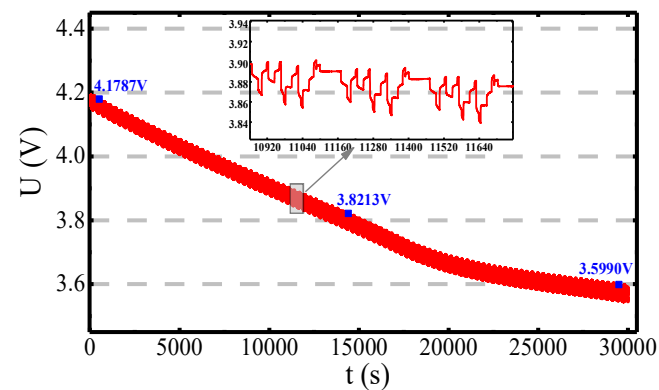

(b): BBDST working condition voltage

Figure 13. BBDST working condition experimental data

Figure 13(a), and (b) are the experimental current and voltage data of the first BBDST working condition, and the experimental current and voltage data of 20 BBDST conditions respectively. Since the BBDST operating condition is power discharge, it can be seen from Figure 13(a) and (b), that as the number of cycles increases, the discharge current increases, and the battery terminal voltage as a whole shows a downward trend. The Thevenin RC modeling was established by parameter identification of HPPC experimental data. To verify the validity of the model, the model data and actual data were compared and analyzed with additional battery operating conditions data. The model was verified using the BBDST working condition, and the various working conditions of the battery were simulated by constant power discharge for a certain period. The test device obtains the current value in the experimental data as the input condition, and the simulated terminal voltage is compared with the experimental acquisition terminal voltage by the simulation model, and the result is shown in Error! Reference source not found.

In Error! Reference source not found.(a), SOC1 is the real terminal voltage data curve obtained by the voltage verification model, and SOC2 (UKF) is the output terminal voltage curve obtained and Error! Reference source not found.(b) is a UKF error by the simulation model under the input current condition. It can be seen from Figure 6 that the simulation and the actual test curves have a similar trend, which can better simulate the battery discharge. According to the established first-order Thevenin RC modeling and the Reduced-order UKF method, the SOC estimation of the BBDST working condition is carried out that can be seen in Figure 13.

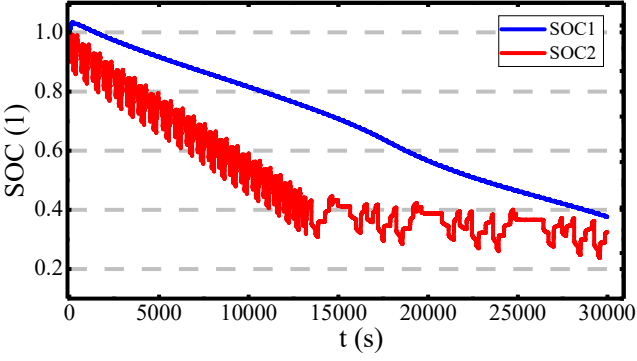

(a): UKF-based on lithium battery SOC estimation effect

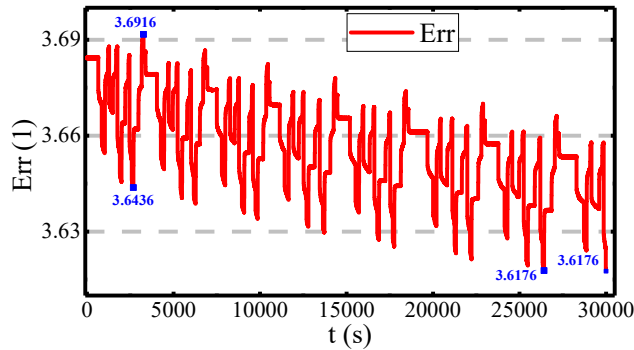

(b): Error-based on lithium battery SOC estimation effect

Figure 14. UKF estimated and Error estimation curves

The SOC estimation results are based on the Reduced-order UKF method proposed. The Reduced-order UKF method estimated lithium-ion battery SOC algorithm was verified by $0.1 \mathrm{C}$ constant current discharge throughout the whole SOC. However, only the change of parameter $R$ has an obvious effect on the model output. This way, the design procedure of model-based adaptive parameter state estimators can then be simplified. The error between the initial value and the true value is given at least $10 \%$ in advance so that the SOC estimation is performed, and the obtained result is shown in Figure 15(a) and (b). 


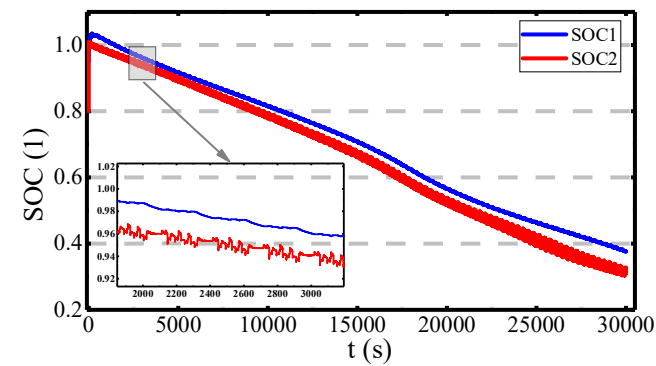

(a): Comparison of SOC estimation curves

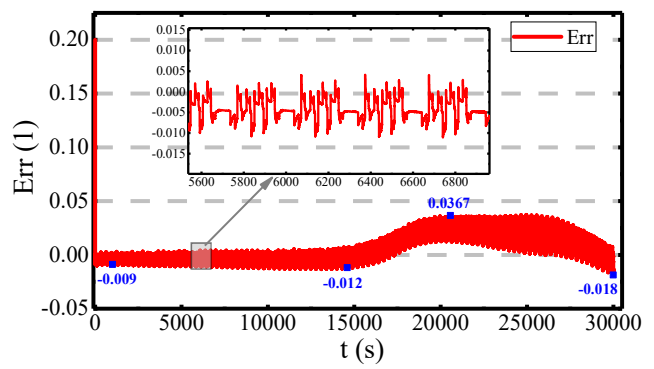

(b): SOC estimation error (ROUKF)

(ROUKF)

Figure 15. ROUKF estimated and Error estimation curves

Figure 15(a) is a graph of estimation results, SOC1 is a true SOC value, and SOC2 is the estimated result for SOC value using a Reduced-order UKF method. Figure 15(b), is an error curve obtained by subtracting two SOC value curves. It is known from Error! Reference source not found. that the error of SOC estimation based on the established Thevenin RC modeling using the Reduced-order UKF is less than $2 \%$. It can correct the error of the initial value very well, does not depend on the accuracy of the initial value, and has a strong correction function.

\section{Conclusions}

It is important and difficult to accurately estimate the SOC of a lithium battery. A battery based on the Thevenin $\mathrm{RC}$ modeling is used, taking into account its advantages of low error, long-term testing, and accounting polarization effects, and transient analysis for power battery charging and discharging. The OCV-SOC and the HPPC method of estimating SOC was analyzed through experiments. From the two approaches, it can be concluded that the OCV-SOC method alone is not an accurate or an independent method to estimate SOC but a basic tool for other methods including the HPPC Reduced-order UKF estimation method. The OCV-SOC is a direct method of measuring the open-circuit voltage and using it to estimate SOC, this resulted in a greater error margin so the advancement of it to the HPPC test method. However, in this method: the use of the Reduced-order UKF method will inevitably introduce nonlinear errors if the nonlinearity of the battery is not established. The Simulink model was established on MATLAB and combined with experimental data under BBDST working conditions for simulation analysis. With the HPPC test and BBDST method, the Thevenin RC modeling was used and its parameters were verified. The results show that the Reduced-order UKF method can estimate the SOC of the lithium battery very well, than the traditional UKF method, and the estimation error can be controlled within $0.3 \%$, which verifies that the Reduced-order UKF method has high accuracy in the SOC estimation of lithium-ion battery. Although the HPPC test with other models has high accuracy than the OCV test, research is still ongoing to get a more accurate algorithm for accurately estimating the SOC of the lithium-ion battery. In future research work, we will be concerned about finding an effective assessment indicator of the State of Health (SOH) of LIB for a safe and sound energy management system of the LIB packs.

\section{References}

1. Garg, A., et al., Design and analysis of capacity models for Lithium-ion battery. Measurement, 2018. 120: p. 114-120.

2. Rohan, R., et al., Melamine-terephthalalde Hyde-lithium complex: a porous organic network based single ion electrolyte for lithium-ion batteries. Journal of Materials Chemistry A, 2015. 3(9): p. 51325139.

3. Juan Pablo Rivera-Barrera 1, Nicolás Muñoz-Galeano 1 ID and Henry Omar Sarmiento-Maldonado 2, SoC Estimation for Lithium-ion Batteries: Review and Future Challenges. Electronics, 2017.

4. Bairwa, B.L., et al., Mars based state of charge estimation using real-life loading condition of lithiumion battery for electric vehicle. AIP Conference Proceedings, 2020. 2294(1): p. 040012.

5. Lijun Zhang, Z.M., Xiangyu Gao, Coupling analysis and performance study of commercial 18650 Lithium-ion batteries under conditions of temperature and vibration. Energies, 2018. 11(10): p. 1-27. 
6. Chen, T., et al., Applications of Lithium-Ion Batteries in Grid-Scale Energy Storage Systems. Transactions of Tianjin University, 2020. 26(3): p. 208-217.

7. Diab, Y., et al., Estimating Lithium-Ion Battery State of Charge and Parameters Using a ContinuousDiscrete Extended Kalman Filter. Energies, 2017. 10(8).

8. Zhang, C., et al., A Generalized SOC-OCV Model for Lithium-Ion Batteries and the SOC Estimation for LNMCO Battery. Energies, 2016. 9(11).

9. Marzetti, S. and F. Mariasiu, Electric vehicle battery technologies: From present state to future systems. Renewable and Sustainable Energy Reviews, 2015. 51: p. 1004-1012.

10. Sun, X., et al., Technology Development of Electric Vehicles: A Review. Energies, 2020. 13(1).

11. Gao, Z.C., et al., State-of-Charge Estimation and Active Cell Pack Balancing Design of Lithium Battery Power System for Smart Electric Vehicle. Journal of Advanced Transportation, 2017. 2017: p. 6510747.

12. Yizhao Gao1, X.Z., Jun Yang1, Bangjun Guo1, Xuan Zhou2, A Novel Model for Lithium-ion Battery Aging Quantitative Analysis Based on Pseudo Two-Dimension Expressions. International Journal of ELECTROCHEMICAL Science Direct, 2019.

13. Sergey $\mathrm{Yu}$ Vassiliev, V.V.S., Eduard E. Levin, Victoria A. Nikitina, Diagnostics of lithium-ion intercalation rate-determining step: Distinguishing between slow desolation and slow charge transfer. Electrochemical Acta, 2019.

14. Bi, Y., Y. Yin, and S.-Y. Choe, Online state of health and aging parameter estimation using a physicsbased life model with a particle filter. Journal of Power Sources, 2020. 476: p. 228655.

15. Liu, X.M. and C.B. Arnold, Effects of Current Density on Defect-Induced Capacity Fade through Localized Plating in Lithium-Ion Batteries. Journal of the Electrochemical Society, 2020. 167: p. 130519.

16. Park, K.-Y., et al., Understanding capacity fading mechanism of thick electrodes for lithium-ion rechargeable batteries. Journal of Power Sources, 2020. 468: p. 228369.

17. Ghannoum, A. and P. Neiva, Graphite lithiation and capacity fade monitoring of lithium-ion batteries using optical fibers. Journal of Energy Storage, 2020. 28: p. 101233.

18. Gao, T., et al., Interplay of Lithium Intercalation and Plating on a Single Graphite Particle. Joule, 2021.

19. Hannan, M.A., et al., Toward Enhanced State of Charge Estimation of Lithium-ion Batteries Using Optimized Machine Learning Techniques. Scientific Reports, 2020. 10(1): p. 4687.

20. Gantenbein, S., et al., Capacity Fade in Lithium-Ion Batteries and Cyclic Aging over Various State-ofCharge Ranges. Sustainability, 2019. 11(23).

21. Rao, M., et al., Investigation of lithium content changes to understand the capacity fading mechanism in LiFePO4/graphite battery. Journal of Electroanalytical Chemistry, 2019. 853: p. 113544.

22. Gao, Y., et al., Lithium-ion battery aging mechanisms and life model under different charging stresses. Journal of Power Sources, 2017. 356: p. 103-114.

23. Barcelona, S. and L. Piegari, Effect of current on cycle aging of lithium-ion batteries. Journal of Energy Storage, 2020. 29: p. 101310.

24. Yang, S., et al., A parameter adaptive method for state of charge estimation of lithium-ion batteries with an improved extended Kalman filter. Scientific Reports, 2021. 11(1): p. 5805.

25. Lv, J., et al., Estimation of the State of Charge of Lithium Batteries Based on Adaptive Unscented Kalman Filter Algorithm. Electronics, 2020. 9(9).

26. Yong Tian1*, R.Y., Jindong Tian1, Shije Zhou1,2 and Chao Hu1,2, Online Estimation of Model Parameters of Lithium-Ion Battery Using the Cubature Kalman Filter. IOP Science, 2017.

27. Zhang, M., K. Wang, and Y.-t. Zhou, Online State of Charge Estimation of Lithium-Ion Cells Using Particle Filter-Based Hybrid Filtering Approach. Complexity, 2020. 2020: p. 8231243.

28. Nassim Noura 1, Loïc Boulon 1 and Samir Jemeï 2,3, A Review of Battery State of Health Estimation Methods: Hybrid Electric Vehicle Challenges. World Electric Vehicle Journal, 2020.

29. Collin, R., et al., Advanced Electric Vehicle Fast-Charging Technologies. Energies, 2019. 12(10). 
30. Yang, X., et al., Parameter Identification of Electrochemical Model for Vehicular Lithium-Ion Battery Based on Particle Swarm Optimization. Energies, 2017. 10: p. 1811.

31. Chen, X., et al., Development of the cycling life model of Ni-MH power batteries for hybrid electric vehicles based on real-world operating conditions. Journal of Energy Storage, 2021. 34: p. 101999.

32. Wang, Y., et al., High-Voltage "Single-Crystal" Cathode Materials for Lithium-Ion Batteries. Energy \& Fuels, 2021. 35(3): p. 1918-1932.

33. P.M.W. Salehen1, M.S.S.a., H. Razali1*, and K. Sopian1,2, Development of battery management systems (BMS) for electric vehicles (EVS) in Malaysia MATEC Web of Conferences 2017.

34. Locorotondo, E., G. Lutzemberger, and L. Puig, State-of-charge estimation based on model-adaptive Kalman filters. Proceedings of the Institution of Mechanical Engineers, Part I: Journal of Systems and Control Engineering, 2020: p. 0959651820965406.

35. Rahbar, O., et al., A novel state of charge and capacity estimation technique for electric vehicles connected to a smart grid based on inverse theory and a metaheuristic algorithm. Energy, 2018. 155: p. 1047-1058.

36. Koehler, U., Chapter 2 - General Overview of Non-Lithium Battery Systems and their Safety Issues, in Electrochemical Power Sources: Fundamentals, Systems, and Applications, J. Garche and K. Brandt, Editors. 2019, Elsevier. p. 21-46.

37. Daniel Juarez-Robles3, Anju Arun Vyas1, Conner Fear4,1, Judith A. Jeevarajan2 and Paratha P. Mukherjee3,1, Over discharge and Aging Analytics of Li-Ion Cells. IOP Science, 2020. 167.

38. Chapter 7 - Lithium-Secondary Cell: Sources of Risks and Their Effects, in Electrochemical Power Sources: Fundamentals, Systems, and Applications, J. Garche and K. Brandt, Editors. 2019, Elsevier. p. 143-266.

39. Dey, S. and B. Ayalew, Real-Time Estimation of Lithium-Ion Concentration in Both Electrodes of a Lithium-Ion Battery Cell Utilizing Electrochemical-Thermal Coupling. Journal of Dynamic Systems, Measurement, and Control, 2017. 139(3).

40. Su, J., et al., An equivalent circuit model analysis for the lithium-ion battery pack in pure electric vehicles. Measurement and Control, 2019. 52(3-4): p. 193-201.

41. Ding, X., et al., An improved Thevenin model of lithium-ion battery with high accuracy for electric vehicles. Applied Energy, 2019. 254: p. 113615.

42. Binqi Zheng 1, Peng Cheng Fu 1,2 ID, Baoqing Li 1 ID and Xiaobing Yuan 1*, A Robust Adaptive Unscented Kalman Filter for Nonlinear Estimation with Uncertain Noise Covariance. Sensoes, 2018. 